\title{
Smartphone-Based Single-Camera Stereo-DIC System: Thermal Error Analysis and Design Recommendations
}

\author{
Liping Yu, Nurlat Bekdullayev, and Gilles Lubineau
}

\begin{abstract}
Coupling a smartphone-based stereo-DIC system using a single smartphone and a 3D-printed four-mirror adapter has been recently proposed by the authors as a costeffective and field-portable 3D displacements measurement tools. However, this system is found to be very sensitive to temperature variation inside the smartphone and the ambient temperature variation around the system, which further can introduce displacement and strain errors into the measured results. Interestingly, the image coordinate drifts of image points that are directly associated with these thermal errors cannot be well explained by the existing temperature-induced camera deformation models. To eliminate the large thermal errors and realize high-accuracy 3D deformation measure-

ment, a comprehensive thermal error analysis is performed for the smartphone-based single-camera stereo-DIC system. The theoretical estimations based on the existing temperature-induced camera deformation model are compared with the experimental observations. Then, all the potential error sources that can introduce thermal errors in the smartphone-based single-camera stereo-DIC system are analyzed and discussed. Several comparison tests confirm that the thermal errors are caused by both the inner structural changes and the deformation from the adapter, and that the thermal errors from the adapter are much larger than those caused by the inner deformation inside the smartphone camera. Finally, a modified mirror adapter was fabricated and proven less sensitive to the temperature variation. This research is expected to explain all the potential thermal error sources in the smartphone-based single-camera stereo-DIC system and further contribute to the correction of the thermal errors and the optimal design of the adapter.
\end{abstract}

Index Terms-Stereo-digital image correlation, smartphone, thermal error.

\section{INTRODUCTION}

$\mathbf{N}$ ON-CONTACT and non-destructive 3D shape, motion and deformation measurements of materials and structures subjected to various external loadings is an important and essential task in characterizing their deformation behavior. Existing methods for this purpose include, but not limited to, electronic speckle pattern interferometry (ESPI) [1], [2], digital shearography (DS) [3], [4], and holographic interferometry (HI) [5], [6], and stereo-digital image correlation [7]-[10] (stereo-DIC). Compared with the interferometric optical techniques (i.e., ESPI, DS and HI), the stereo-DIC technique has the advantages of simple experimental setup, wide range of applicability and low environmental vulnerability. With the

Manuscript received August 25, 2020; accepted January 20, 2021 This work was supported by the King Abdullah University of Science and Technology (KAUST) under Award BAS/1/1315-01-01. The associate editor coordinating the review of this article and approving it for publication was Prof. Zeljko Ignjatovic. (Corresponding author: Gilles Lubineau.)

The authors are with the COHMAS Laboratory, Physical Sciences and Engineering Division (PSE), King Abdullah University of Science and Technology (KAUST), Thuwal 23955-6900, Saudi Arabia (e-mail: liping.yu@kaust.edu.sa; gilles.lubineau@kaust.edu.sa).

Digital Object Identifier 10.1109/JSEN.2021.3054805 continuous developments and massive applications in recent years, stereo-DIC has been proven to be a powerful noncontact technique for measuring the full-field 3D displacement and strain field of both planar and curved objects, used in fields as diverse as materials science [11]-[13], biomechanics [14]-[16], composite materials [17], [18], high-speed deformation measurement [19]-[21], and many other fields.

Usually, the use of a regular binocular stereo-DIC system is discouraged by the requirement of two industrial-grade cameras and the stringent synchronization between these two cameras. Aiming at a simple, cost-effective and field-portable $3 \mathrm{D}$ deformation measurement tool, we recently established a smartphone-based stereo-DIC system inspired by the mirrorbased single-camera stereovision technique [22]-[24]. This system can accurately measure $3 \mathrm{D}$ shape and deformation on the object surface by only using a single smartphone and a 3D-printed four-mirror adapter. Compared with the conventional stereo-DIC system using two synchronized cameras, the smartphone-based single-camera stereo-DIC system is more cost-effective, compact and field-portable. First, the cost of the imaging cameras and camera synchronization can be greatly reduced or even neglected, as the smartphone is 
(a)

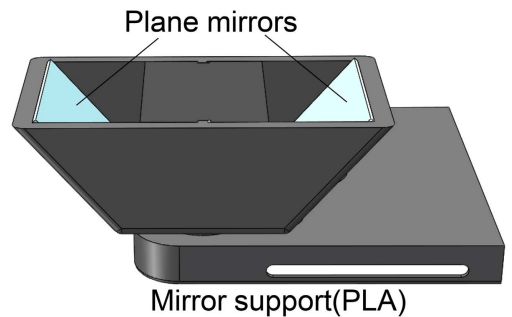

(b)

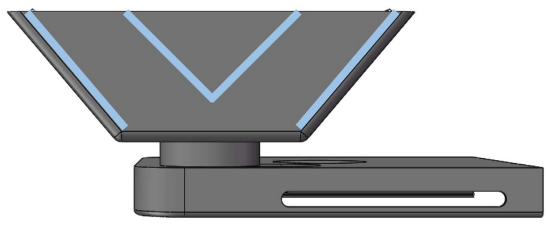

(c)

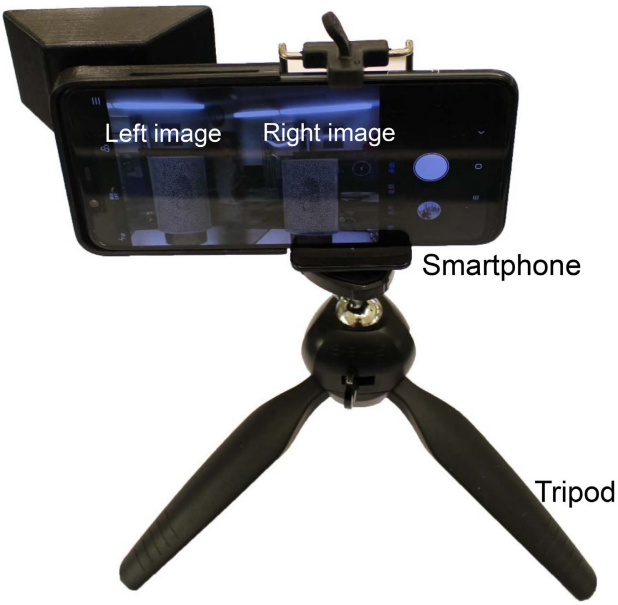

Fig. 1. (a, b) Model of the four-mirror adapter in two different views and (c) the established smartphone-based single-camera stereo-DIC system.

ubiquitous now. Then, the smartphone can capture the image, process image and transmit data whilst requiring little space and being independent of external power, making the stereoDIC system extremely compact and field-portable. Moreover, after booming developments in recent years, smartphones can offer users high-quality digital cameras, large storage capacities, significant computational power, and user-friendly software. For example, the performances of the smartphone camera are being dramatically improved by the manufactures in terms of the effective focal length, image resolution and frame rate. The maximum image resolution can reach tens of millions of pixels, and a maximum frame rate of 960 frames per second can be realized by state-ofthe-art smartphones. Owing to the attractive features of the smartphones, the smartphone-based stereo-DIC system has significant potential to be used for $3 \mathrm{D}$, motion and deformation measurement, especially in resource-limited institutions and field settings.

However, it is found that the established smartphone-based single-camera stereo-DIC system is extremely sensitive to the temperature variation inside the smartphone and the ambient temperature variation around the system [25]. These temperature variations further can introduce considerable displacement and strain errors into the measured results. According to our recent investigation [25], [26], the calculated displacement and strain errors in stereo-DIC are determined by the temperature-dependent disparity (i.e., the difference between image coordinates of two projection points) changes while the camera parameters are assumed to be constant. Previously, the temperature-dependent disparity changes are considered to be caused by the inner structure changes of the camera, e.g., the translation and/or rotation of camera lens and sensor. However, our recent study indicates that the temperaturedependent disparity changes are not only introduced by the inner structure changes of the camera, but also from the thermal deformation of the four-mirror adapter. To eliminate the large thermal errors and realize high-accuracy 3D deformation measurement, comprehensive thermal error analysis for the smartphone-based single-camera stereo-DIC system is required.

In this paper, two different models, i.e., a virtual camera model and a virtual object model are proposed to understand the imaging model of the single-camera stereovision system. Based on the imaging model, the theoretical estimations based on the existing temperature-induced camera deformation model and the experimental observation of the image coordinate drifts are compared. Then, all the potential error sources that can introduce thermal errors in the smartphonebased single-camera stereo-DIC system are analyzed and discussed. Several comparison tests confirm that the thermal errors are caused by both the inner structural changes and the deformation from the adapter, and the thermal errors from the adapter are much larger than those caused by the inner deformation inside the smartphone camera. Finally, a modified mirror adapter was fabricated and proven less sensitive to the ambient temperature variation.

\section{SmartPhone-Based Single-Camera STEREO-DIC SYSTEM}

\section{A. System Design}

Figure 1 shows the established smartphone-based singlecamera stereo-DIC system, which is composed of a single smartphone (Mi8, Xiaomi, Inc., Beijing, China) and a fourmirror adapter. The physical focal length of the used camera lens is measured as $5.2 \mathrm{~mm}$. The four-mirror adapter consists of four plane mirrors, a 3D-printed mirror support (material: Polylactic Acid, PLA) and a 3D-printed smartphone shell (material: PLA). Two interior mirrors $(35 \mathrm{~mm} \times 20 \mathrm{~mm} \times$ $1 \mathrm{~mm}$ ) are fixed on two sides of the inner mirror support, while two exterior mirrors $(35 \mathrm{~mm} \times 30 \mathrm{~mm} \times 1 \mathrm{~mm})$ are glued tightly on the outside support. Note that the size and inclination angle of the mirrors need to be chosen on a case by case basis according to the focal length and sensor size of the smartphone camera [27], [28]. For example, larger mirrors are required for a smartphone with a small focal length. As shown in Fig. 1(c), the four-mirror adapter can be well coupled 


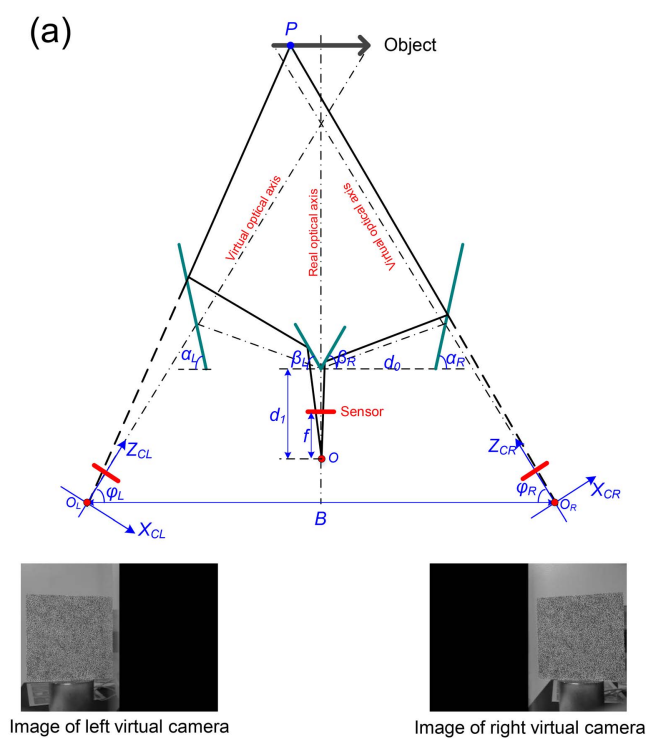

(b)

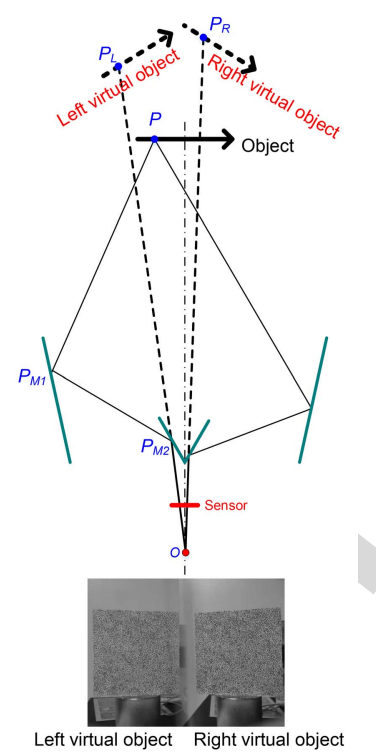

Fig. 2. Two imaging models for the single-camera stereo-DIC system: (a) virtual camera model (b) virtual object model.

TABLE I

System Parameters of the Single-Camera Stereo-DiC System and the Corresponding Intrinsic and Extrinsic Parameters. Note That the Left Two Mirrors Are Assumed Symmetric to the Right Ones About the Optical Axis

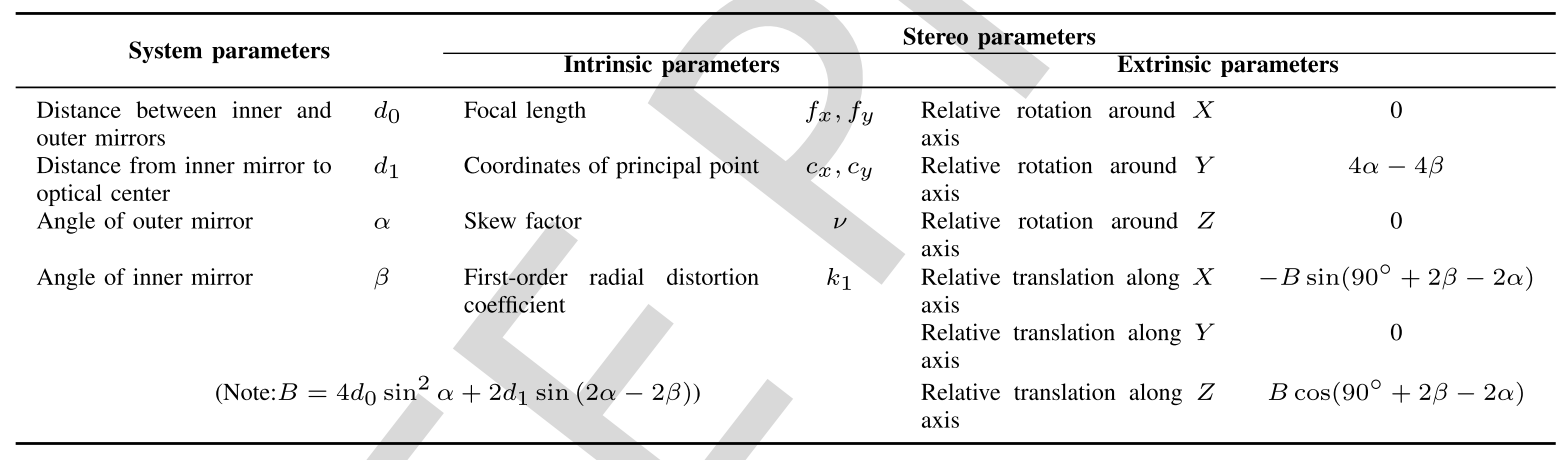

with the smartphone, thus making the system extremely fieldportable. With the aid of the four-mirror adapter, two views of the object surface are projected onto the left and right half sensor through two different optical paths. By capturing and processing a set of calibration images and object images, the 3D shape and deformation on the object surface can be retrieved. The detailed process of the 3D measurement has been described in previous researches [25], thus is not introduced here.

\section{B. Virtual Camera Model and Virtual Object Model}

To better understand the established smartphone-based single-camera stereo-DIC system, here we present two imaging models for this system, i.e., virtual camera model and virtual object model. Fig. 2 (a) shows the schematic diagram of the virtual camera model of the single-camera stereo-DIC system. With the aid of the four reflection mirrors, a point $P$ on the object can be split and projected onto the left and right halves of the camera sensor. By rearranging the optical path and optical axis through the law of reflection, this system can be seen as a pseudo stereo-DIC system using two virtual cameras. The intrinsic parameters of this pseudo stereo-DIC system are determined by the camera parameters (i.e., focal length $(f)$ and position of the principal point in $x$ and $y$ directions $\left(c_{x}\right.$ and $\left.\left.c_{y}\right)\right)$.

The extrinsic parameters are highly related with the structural parameters, i.e., the horizontal inclination angle of the mirrors $\left(\alpha_{L}, \alpha_{R}, \beta_{L}, \beta_{R}\right)$, the distance between the optical center to the inner mirror $\left(d_{1}\right)$ and the distance between the left and right mirrors $\left(d_{0}\right)$. Note that the left two mirrors are assumed to be symmetric to the right ones here. The extrinsic parameters can be regarded as the relative rotation and translation between the left and right cameras. For the pseudo stereo-DIC system shown in Fig. 2(a), the relative rotation angles between two virtual cameras can be determined as $\left(0, \pi-\phi_{L}-\phi_{R}, 0\right)$ around the three axes, and the relative translation is expressed as $\left(-B \sin \phi_{R}, 0, B \cos \phi_{R}\right)$.

Here, $\phi_{L}$ and $\phi_{R}$ are the inclination angles between the virtual optical axes and the baseline $\left(O_{L} O_{R}\right)$, and $B$ is the baseline distance. Table I summarizes the structural parameters 

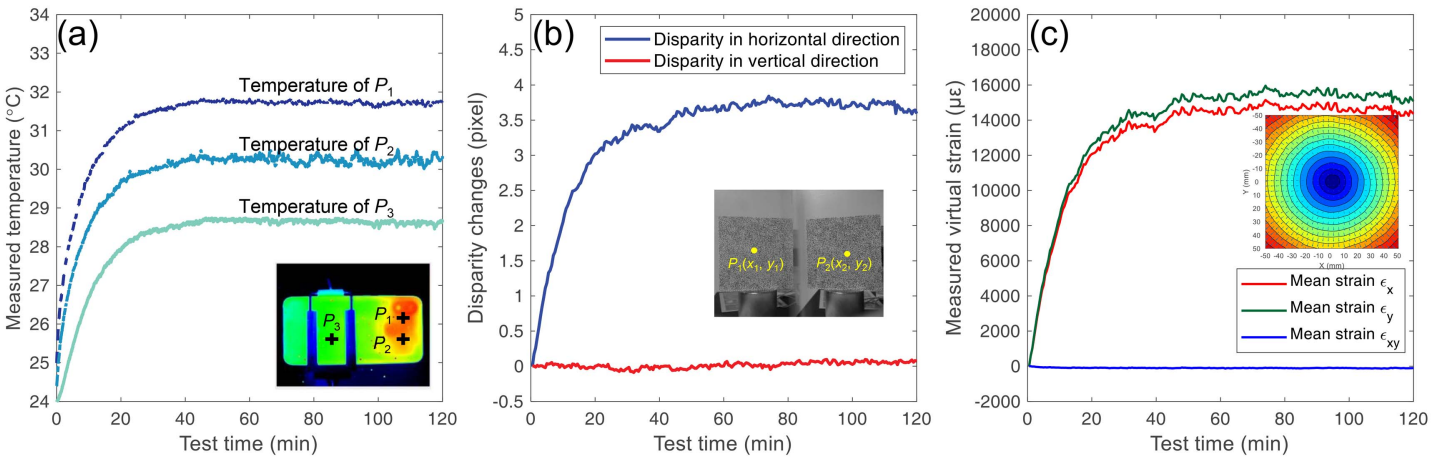

Fig. 3. (a) Measured temperatures as a function of the test time at different points of the smartphone back surface, (b) the disparity changes between the two projections of a point on the object, (c) the measured thermal strain errors as a function of the test time.

and the corresponding intrinsic and extrinsic parameters of the single-camera stereo-DIC system. Once the structural parameters are determined and the camera was pre-calibrated, we can determine all the intrinsic and extrinsic parameters of the pseudo stereo-DIC system. Some geometric relationships can be found in the existing researches [27], [28].

The imaging model of the single-camera stereo-DIC system can also be described by a virtual object model. When placing a four-mirror adapter before the camera, an object point $P$ will be offset from its actual position and be viewed as two virtual points $P_{L}$ and $P_{R}$. As shown in Fig. 2(b), suppose $P_{M 1}$ and $P_{M 2}$ are the reflection points of the left optical path on the left outer mirror and left inner mirror, the position of the left virtual point can be determined by extending the optical path $O P_{M 2}$ to $O P_{L}$. The length of $P_{M 2} P_{L}$ is equal to the sum of $P_{M 2} P_{M 1}$ and $P_{M 1} P$. Once the four-mirror adapter is fixed, the positions and orientations of the virtual objects will be also fixed. Actually, the virtual camera model is equivalent to the virtual object model. By moving the optical center of the virtual cameras $\left(O_{L}\right.$ and $\left.O_{R}\right)$ to the position of the real optical center $(O)$ and making the virtual optical axis and the real optical axis coincided, the virtual camera model can be transformed into the virtual object model.

\section{THERMAL ERROR ANALYSIS OF SMARTPHONE-BASED SINGLE-CAMERA STEREO-DIC SYSTEM}

\section{A. Thermal Errors}

As shown in Fig. 3, considerable thermal displacement and strain errors were found in the established smartphonebased stereo-DIC system during a static test. These thermal errors are highly related to the measured temperature on the smartphone back surface. According to our recent investigation [26], the thermal errors in a conventional stereo-DIC system can be well explained by a real model and a calculation model.

The calculated displacement and strain errors in stereoDIC are determined by the temperature-dependent disparity (i.e., the difference between image coordinates of two projection points) changes while the camera parameters are assumed to be constant. Fig. 3(a) shows the measured temperatures on the smartphone back surface as a function of the test time. The temperature variation will change the disparity (a)

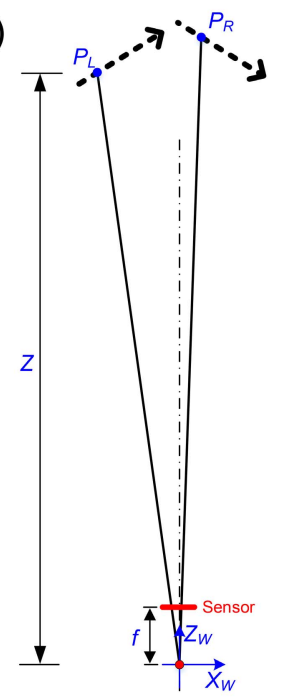

(b)

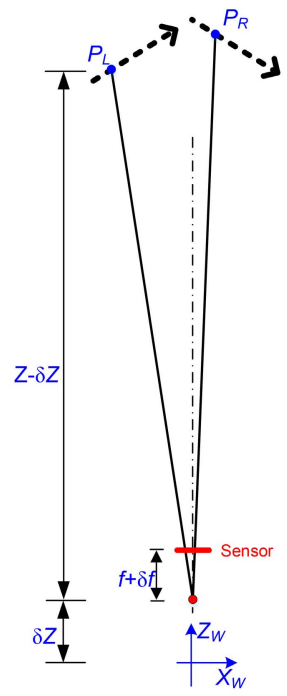

Fig. 4. Imaging model based on the virtual object model: (a) before camera self-heating, (b) after camera self-heating.

between the left and right images, which further leads to considerable strain errors shown in Fig. 3(c). Note that similar trends of the back surface temperatures were observed from the repeated tests with temperature increment measured as about 6-10 degrees celsius, but the initial temperature may depends on the initial state of the smartphone. As shown in Fig. 3(b) and (c), the normal strain errors are almost in linear relationships with the horizontal disparity changes. To fully understand and eliminate these thermal errors, it is of great importance to determine all the possible error sources that may change the disparity between image coordinates of two projection points.

\section{B. Theoretical Estimation of the Image Coordinate Drift}

Existing researches show that the out-of-plane translations (along the optical axis direction) of camera lens and sensor are the dominant consequences of the camera self-heating [29]-[31]. Fig. 4(a) shows the imaging model of the system based on the virtual object model before the camera selfheating. Here, we define the world coordinate system at the camera center, and assume the 3D coordinates of a virtual 

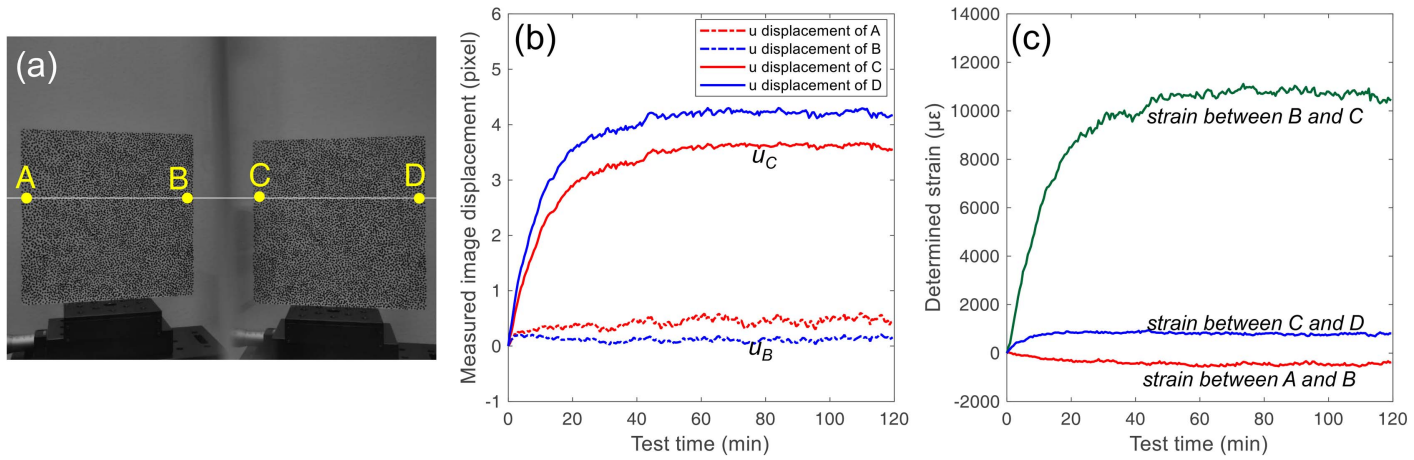

Fig. 5. (a) An image captured by the smartphone during the self-heating test, (b) the u displacements of four points of interest, (c) the strain values between these points.

${ }_{236}\left[\begin{array}{c}x \\ y \\ 1\end{array}\right]=\frac{1}{Z}\left[\begin{array}{ccc}\frac{f}{d_{x}} & 0 & c_{x} \\ 0 & \frac{f}{d_{y}} & c_{y} \\ 0 & 0 & 1\end{array}\right]$. written as, plane can be determined as, object point in the world coordinate system are $(X, Y, Z)$. Then the pinhole model for this point can be expressed as,

$$
\left(\left[\begin{array}{lll}
1 & 0 & 0 \\
0 & 1 & 0 \\
0 & 0 & 1
\end{array}\right]\left[\begin{array}{l}
X \\
Y \\
Z
\end{array}\right]+\left[\begin{array}{l}
0 \\
0 \\
0
\end{array}\right]\right)
$$

where $(x, y)$ are the image coordinate of this point, $f_{x}=f / d_{x}$ and $f_{y}=f / d_{y}$ are focal lengths in $x$ and $y$ directions ( $f$ is the physical focal length of the lens, $d_{x}$ and $d_{y}$ are scale factors relating pixels to distance in both directions), $c_{x}$ and $c_{y}$ are the coordinates of the principal point on image plane.

As shown in Fig. 4(b), if camera center has an out-ofplane translation $\delta Z$ along the optical axis, the focal length has a small change $\delta f$ and the principle point has a drift $\left(\delta c_{x}, \delta c_{y}\right)$, the pinhole model for the virtual object point can be

$$
\begin{aligned}
& {\left[\begin{array}{c}
x^{\prime} \\
y^{\prime} \\
1
\end{array}\right]=\frac{1}{Z-\delta Z}\left[\begin{array}{ccc}
\frac{f+\delta f}{d_{x}} & 0 & c_{x}+\delta c_{x} \\
0 & \frac{f+\delta f}{d_{y}} & c_{y}+\delta c_{y} \\
0 & 0 & 1
\end{array}\right] . } \\
&\left(\left[\begin{array}{lll}
1 & 0 & 0 \\
0 & 1 & 0 \\
0 & 0 & 1
\end{array}\right]\left[\begin{array}{c}
X \\
Y \\
Z
\end{array}\right]+\left[\begin{array}{c}
0 \\
0 \\
-\delta Z
\end{array}\right]\right)
\end{aligned}
$$

Then, the in-plane displacements of this point on the sensor

$\left[\begin{array}{l}u \\ v\end{array}\right]=\left[\begin{array}{l}x^{\prime} \\ y^{\prime}\end{array}\right]-\left[\begin{array}{l}x \\ y\end{array}\right] \approx\left[\begin{array}{l}\left(\frac{\delta f}{f}+\frac{\delta Z}{Z}\right)\left(x-c_{x}\right)+\delta c_{x} \\ \left(\frac{\delta f}{f}+\frac{\delta Z}{Z}\right)\left(y-c_{y}\right)+\delta c_{y}\end{array}\right]$

where $\left(x-c_{x}\right)$ and $\left(y-c_{y}\right)$ are the distance from the image point to the image center. It is clear from Eq. 3 that the normal strains $(\partial u / \partial x, \partial v / \partial y)$ are determined by the term $(\delta f / f+\delta Z / Z)$. For all the object points, the values of this term are close. This means that an even expansion or compression deformation will be introduced by the out-of-plane translation of the sensor and camera lens. Note that the in-plane translations of the object will equally introduce in-plane translations on the left and right sensor. As a result, the disparity caused by the in-plane translation should be zero. Based on the triangulation principle, the caused error will be zero.

\section{Thermal Error Analysis}

Figure 5 (a) shows an image captured by the smartphonebased single-camera stereo-DIC system during the self-heating test. Four horizontal points are selected on the image as points of interest. Fig. 5(b) shows $u$ displacements of these four points as a function of test time. Although the smartphone and the object were both kept static, the displacements of these points vary with the test time. It is clear that the displacements of points $C$ and $D$ are much larger than those of $A$ and $B$. The strain between points $B$ and $C$ are larger than those on the left and right images. These observations indicate that the left and right image have a rigid separation, but not a uniform deformation. However, according to Eq.(3), the $u$ displacement of point $B$ and $C$, and the strains between these points should be close. The large inconsistency between the experimental observations and theoretical estimations indicate that the temperature-dependent image coordinate drift cannot be well explained by the assumed model, and are caused by the other factors.

Figure 6 lists all the possible error sources in the established smartphone-based stereo-DIC system that will change the disparity between the two projections. The possible errors can be separated into two parts, i.e., errors from the smartphone and errors from the adapter. The error sources from the smartphone mainly include out-of-plane translations and rotations of the camera lens and/or camera, while the in-plane translations will not cause the disparity changes. According to the aforementioned analysis, both the out-of-plane translations and rotations of the camera lens and camera will not lead to a rigid separation of the left and right images. The major disparity change can only be attributed to unfavorable factors from the adapter. A careful inspection of the four-mirror adapter indicates that the most possible error source is the thermal deformation of the mirror support. Any deformation of the mirror support will slightly change the inclination angle of the mirrors. As shown in Fig. 2(a), the change of the 


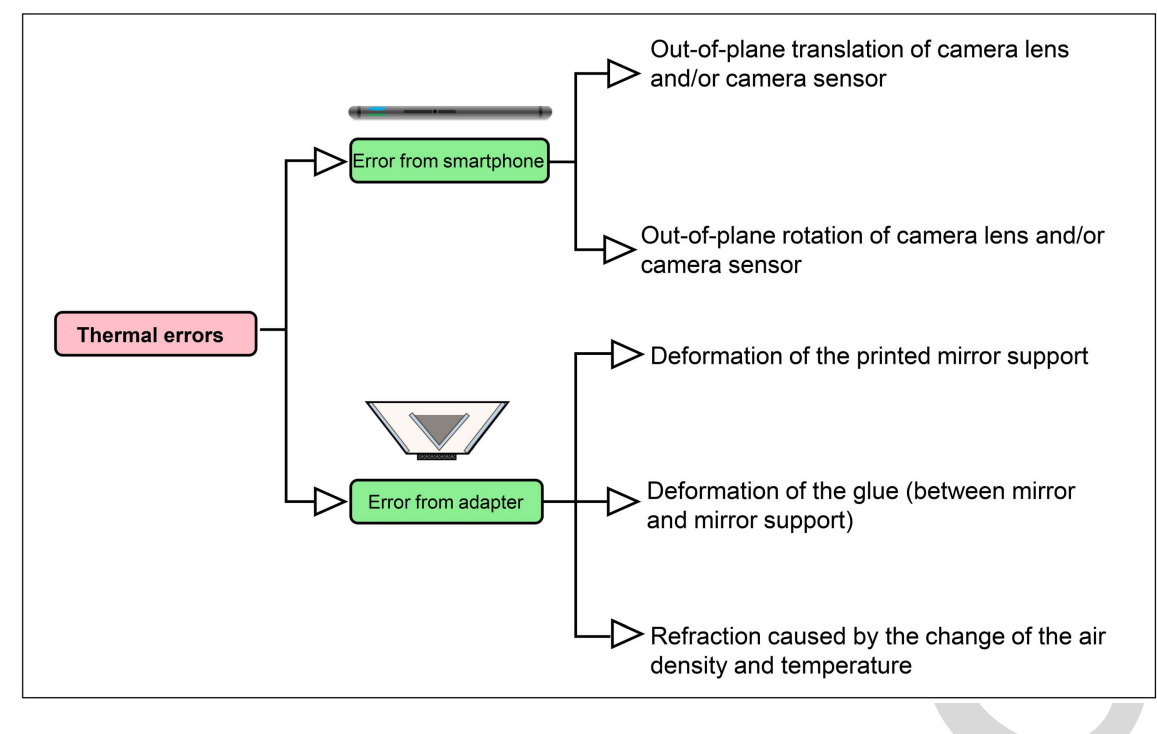

Fig. 6. All the possible error sources that may cause the disparity changes between the two projections.
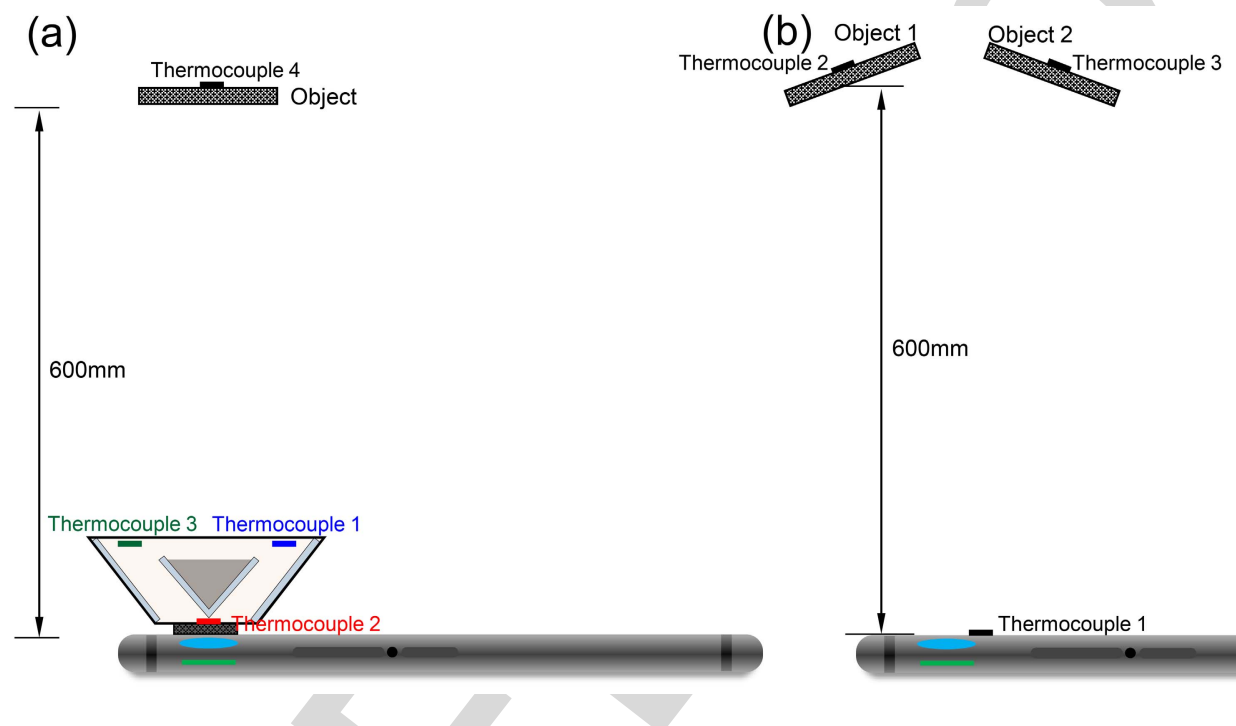

inclination angle of the mirror will slightly rotate the virtual optical axes. The rotation of the virtual optical axis will cause a rigid translation of the left and right images, further leads to the separation of the two images.

\section{A. Experimental Details}

\section{EXPERIMENTS}

To investigate the error sources and verify the correctness of the above analysis, a series of experiments were performed. As shown in Fig. 1(c), the established smartphone-based stereo-DIC system is mainly composed of an Android-based smartphone (Mi8, Xiaomi, Inc., Beijing, China), a homemade four-mirror adapter and a small tripod. Note that here we used the conventional front surface reflective mirror for the adapter, which is negligible in cost. The smartphone is equipped with two imaging lenses $(f / 1.8, f / 2.4)$ and two Sony IMX363 sensors $(4032 \times 3024$ pixels). During the test, only one back camera $(f / 2.4)$ of the smartphone was used for image acquisition. The details about the two sets of 317 experiments are summarized as follows.

1) Comparison Test 1: self-heating tests with and without the adapter were performed. Figure 7 (a) shows the experimental arrangement of the camera self-heating test with the adapter. A rectangular plate with a physical size of $80 \mathrm{~mm} \times 80 \mathrm{~mm}$ was placed in front of the system. With the aid of the four-mirror adapter, two virtual objects were generated and projected onto the left and right halves sensor. In this configuration, the disparity between the left and right virtual images will be changed by the deformation inside the smartphone camera and the small changes from the adapter, as no external loading was applied to the plate. Then, we remove the adapter, and then placed two rectangular plates of similar size in front of the smartphone according to the virtual object model in Fig. 2(b). Under this configuration, the two objects will be projected onto the left and right halves sensor. The relative movement between the two projections can only be attributed 

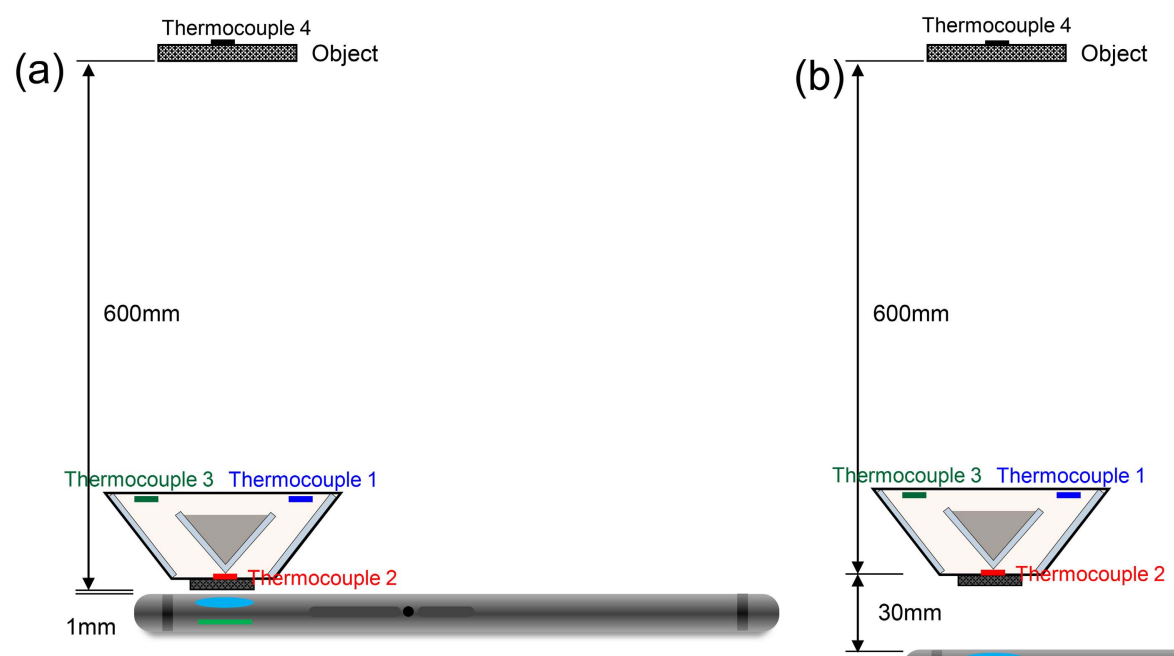

Fig. 8. Experimental arrangement of the camera self-heating tests with separated adapter: (a) spacing $=1 \mathrm{~mm}$, (b) $\mathrm{spacing}=30 \mathrm{~mm}$.

to the deformation inside the smartphone camera. Also, this relative movement can be regarded as the virtual disparity change. In these two experiments, the smartphone and the plates were kept static. Surface images of the plates were automatically recorded by the smartphone every 30 seconds. The recording process lasted for 2 hours, resulting in about 240 images recorded in each test. The first recorded image was adopted as the reference image, and the remaining images were considered as the deformed images. To monitor the temperature variation of the smartphone system during the test, several thermocouples were attached onto the system.

2) Comparison Test 2: self-heating tests with separated adapter. Figure 8 shows the experimental arrangement of the camera self-heating tests with the separated adapter. In the first configuration, the adapter was slightly separated from the smartphone, which is similar to Fig. 7(a) but with some minimal distance gap. Then, we increased the distance between the adapter and smartphone, to alleviate the heating effect of the smartphone on the adapter. The only difference between these two configurations is the small change in the virtual baseline distance. Similarly, images were recorded by the smartphone every 30 seconds. The recording process lasted for 2 hours, resulting in about 240 images recorded in each test. Before the test, a set of calibration images were captured for both tests.

\section{B. Image Processing}

In the first test, the images captured during the test with adapter were exported to computer and processed using the method well described in Ref. [25], which is a stereo-DIC method essentially. After the image processing, the images coordinates of all the calculation points in the left image and the corresponding coordinates of the points in the right image were determined. Then the disparity data of all the calculation points can be calculated. For the test without adapter, we first chose the center points of the two plates as the points of interest, and then calculated the displacements of these points using a self-developed point tracking algorithm
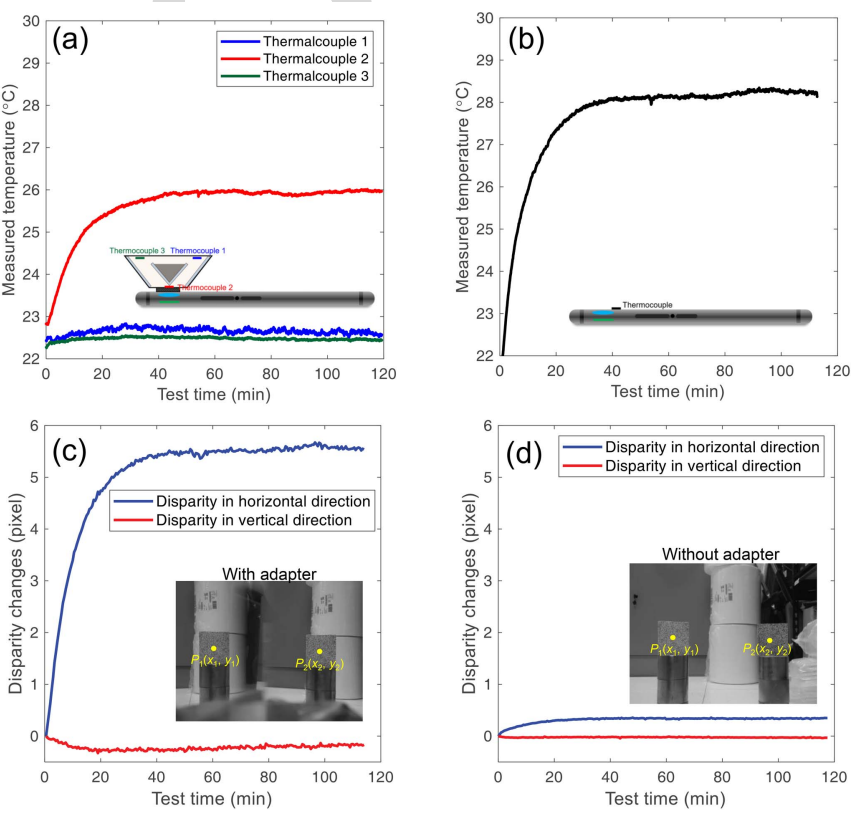

Fig. 9. (a, b) Measured temperatures of the thermocouples as a function of test time for the self-heating tests with and without the adapter, (c) the disparity changes of an object point as a function of test time, (d) the relative movement between the left and right images(virtual disparity change).

(based on 2D-DIC). We consider the difference between the coordinates of these two points as the virtual disparity. In the second test, we processed all the images using the method in [25]. As a result, the virtual displacements and strains caused by the thermal errors can be retrieved.

\section{Results}

1) Comparison Test 1: Figure 9 (a) and (b) show the measured temperatures of the thermocouples as a function of test time for the self-heating tests with and without the adapter. For the test with the adapter, it is observed that the temperature 

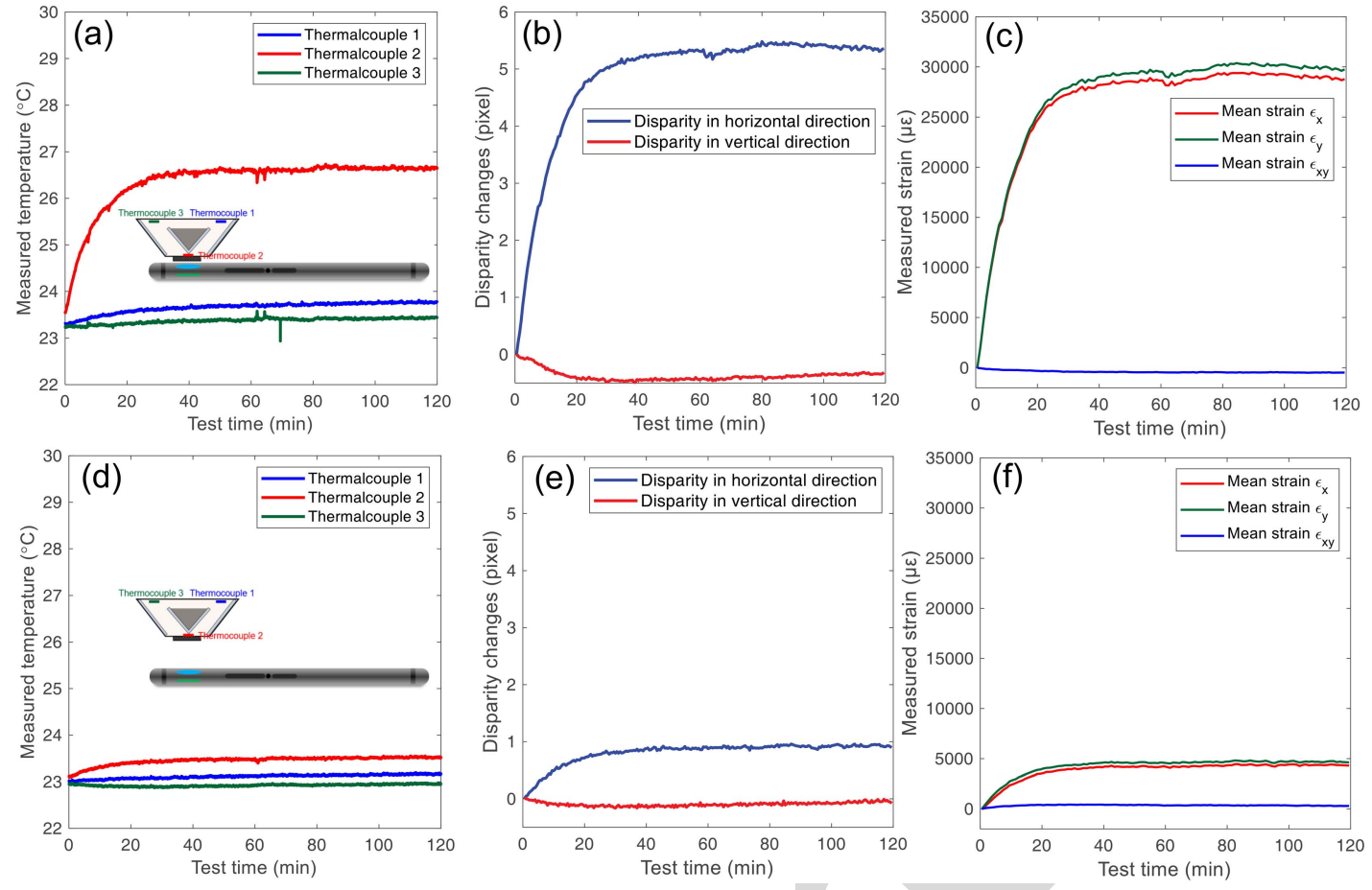

Fig. 10. (a, d) Measured temperatures of the thermocouples as a function of test time for the self-heating tests with the separated adapters, $(b, e)$ the disparity changes of an object point as a function of test time for the two tests, (c, f) the mean thermal strain errors as a function of test time.

of thermocouple 2 first increased to 26 degree celsius and then stabilized at this temperature, while those of the other two thermocouples were almost not changed. This observation indicates that the adapter experienced an apparent temperature gradient during the test. As a result, any small deformation of adapter and deformation inside the smartphone will cause the movement and deformation of the projected images, changing the disparities between the left and right images. As shown in Fig. 9(c), the $x$-directional disparity of an object point presents a similar trend with the temperature variations. The maximum $x$-directional disparity change is determined as about 5.5 pixels, while the $y$-directional disparity almost keeps constant. When the adapter was removed, a similar temperature variation was also observed on the back surface of the smartphone. As this thermocouple was directly attached to the smartphone back surface, the maximum temperature is higher than those of the three thermocouples in the comparison test. Then, we calculated the image displacements of two points on the left and right objects and their relative movements. Because the two objects in this test are not correlated, here we regard the relative movement as the virtual disparity change, which reflects the deformation inside the smartphone. The relative movement of the two points shown in Fig. 9(d) presents a similar trend with the comparison test, but the maximum $x$-directional relative movement is much smaller than that of the test with the adapter. It is therefore concluded that the existence of an adapter will greatly change disparity, and thermal error from the adapter is larger than that from the deformation inside the smartphone.

2) Comparison Test 2: Figure 10 (a) and (d) show the measured temperatures of the thermocouples as a function of test time for the self-heating tests with the separated adapters.
It is clear that the temperature variation of the thermocouple is smaller when the adapter is much separated from the smartphone. As a result, the disparity variation between the two corresponding points is quite different. As shown in Fig. 10(b) and (e), the disparity variation when the adapter is close to the smartphone is much larger than that in the comparison test. If the thermal error from the adapter is neglected, the disparity variation should be very close. By processing the captured images of these two tests, the thermal displacement and strain errors can be determined. Fig. 10(c) and (f) show the mean in-plane strain as a function of test time for the two tests with the adapter. As the smartphone and object were kept static, these measured strains can be regarded as thermal strain errors. As shown in Fig. 10(c) and (f), the determined strain errors are highly correlated with the disparity changes. The maximum normal strain errors when the adapter is close to the smartphone are determined as about 30000 microstrains, which is much larger than the comparison test. This comparison test further confirms that the thermal errors from the adapter are much larger than those from the deformation inside the smartphone.

\section{ERROR ELIMINATION THROUGH STRUCTURAL OPTIMIZATION}

According to the mechanism of thermal error generation, the measured thermal errors are determined by the unfavorable disparity changes between the left and right images due to the thermal deformation of four-mirror adapter and camera structures. Due to the small baseline and focal length, the smartphone-based single-camera stereo-DIC system is usually very sensitive to the unfavorable disparity changes. Therefore, we should eliminate the disparity changes caused 

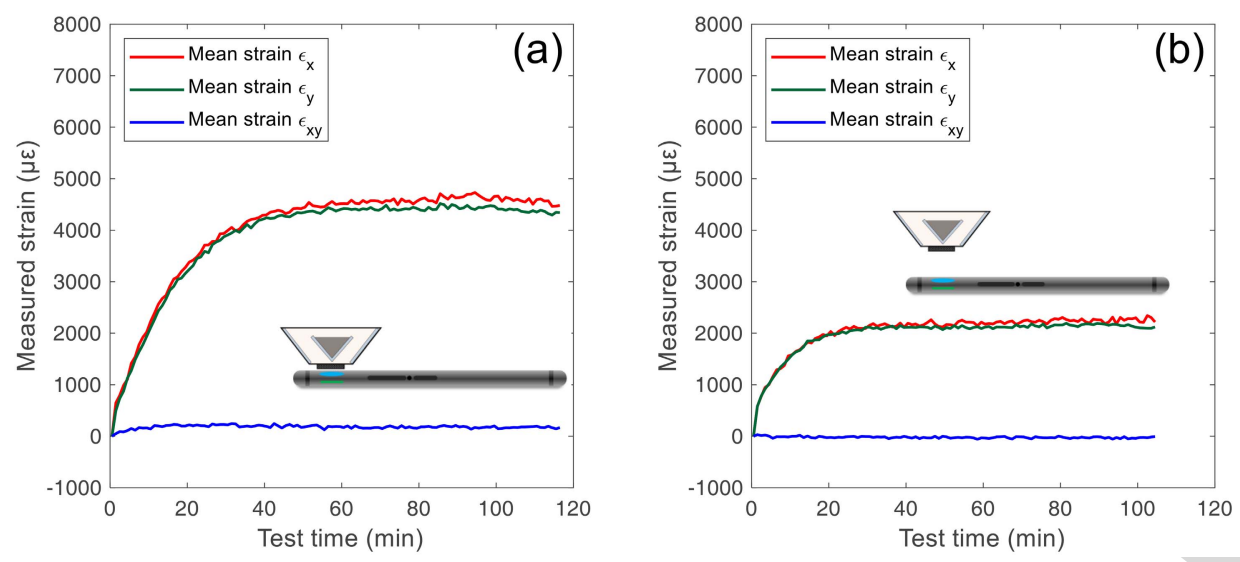

Fig. 11. The mean thermal strain errors as a function of test time when using a steel mirror adapter: (a) spacing $=1 \mathrm{~mm}$, (b) spacing $=30 \mathrm{~mm}$.

TABLE II

Comparison of System Parameters and Maximum Errors Among Four Tests Using Two Different Adapters

\begin{tabular}{|c|c|c|c|c|}
\hline & Configuration a & Configuration b & Configuration $\mathrm{c}$ & Configuration d \\
\hline Material of adapter & PLA & PLA & steel & steel \\
\hline $\begin{array}{l}\text { Thermal expansion coefficient of the } \\
\text { material }\end{array}$ & $68 \times 10^{-6} /{ }^{\circ} \mathrm{C}$ & $68 \times 10^{-}$ & $11-13 \times 10^{-6} /{ }^{\circ} \mathrm{C}$ & $11-13 \times 10^{-6} /{ }^{\circ} \mathrm{C}$ \\
\hline $\begin{array}{l}\text { Distance between smartphone and } \\
\text { adapter }\end{array}$ & $1 \mathrm{~mm}$ & $30 \mathrm{~mm}$ & $1 \mathrm{~mm}$ & $30 \mathrm{~mm}$ \\
\hline Virtual baseline distance & $46 \mathrm{~mm}$ & $56 \mathrm{~mm}$ & $41 \mathrm{~mm}$ & $49 \mathrm{~mm}$ \\
\hline $\begin{array}{l}\text { Pan angle between the two virtual cam- } \\
\text { eras }\end{array}$ & $28.3^{\circ}$ & $27.8^{\circ}$ & $22.7^{\circ}$ & $22.8^{\circ}$ \\
\hline Working distance & $600 \mathrm{~mm}$ & $600 \mathrm{~mm}$ & $600 \mathrm{~mm}$ & $600 \mathrm{~mm}$ \\
\hline Maximum normal strain errors & $30000 \mu \epsilon$ & $5000 \mu \epsilon$ & $4200 \mu \epsilon$ & $2000 \mu \epsilon$ \\
\hline
\end{tabular}

by the deformation of adapter as possible when changing the size of the adapter is not first option. For this purpose, we can optimize the four-mirror adapter by using the material with a low thermal expansion coefficient or separating the smartphone and the adapter as possible. To verify the effectiveness of these optimizations, we fabricated a four-mirror adapter of the same size using steel and performed two self-heating tests. Note that the size and thickness of this steel adapter are same with the previous one made of PLA. The experimental arrangements of these two tests are same with those shown in Fig. 8. By processing the acquired images, the thermal strain errors can be retrieved.

Fig. 11(a) and (b) show the measured mean in-plane strain errors as a function of test time for the two tests using the steel adapter. Compared with the tests using the PLA adapter, the strain errors present similar trends but the maximum strains greatly decrease. As shown in Fig. 11 (a) and (b), the maximum normal strain errors when the adapter is close to the smartphone are determined as about 4000 microstrains, which is nearly twice as large as those when the smartphone and the adapter are separated. These observations show that the thermal errors in the smartphone-based single-camera stereoDIC system are caused by both the thermal deformation inside the smartphone and the thermal deformation of the fourmirror adapter, confirming the correctness of the previous analysis. Table II lists the system parameters and maximum errors among four tests using two different adapters. The main extrinsic parameters (pan angle, baseline distance) of two virtual cameras in these four configurations are close, while the maximum strain error varies greatly. It is clear that the use of a steel greatly decreases the thermal strains.

\section{CONCLUSION}

This paper focuses on thermal error analysis and structural optimization of the recently established smartphonebased single-camera stereo-DIC system. Two different imaging models including the virtual camera model and virtual object model are first proposed to understand the optical path of the system. Then, the theoretical image coordinate drift that will cause thermal errors are estimated based on the existing camera deformation model. The inconsistency between the theoretical estimations and the experimental observations indicates that the thermal errors are caused by both the inner structural changes and the thermal deformation from the adapter. Experimental results from two sets of comparison tests verify these analyses and show that the thermal errors from the adapter are much larger than those caused by the inner deformation inside the smartphone camera. Finally, based on the thermal error analysis, a modified mirror adapter was fabricated and proven less sensitive to the ambient temperature variation. This research is expected to explain all the potential thermal error sources in the smartphone-based single-camera stereo-DIC system and further contribute to the correction of the thermal errors and the optimal design of the adapter. Also, we expect to couple this system with a self-developed smartphone application based on a parametric model in our following works. 


\section{REFERENCES}

[1] A. Martínez, J. A. Rayas, R. Rodríguez-Vera, and H. J. Puga, "Threedimensional deformation measurement from the combination of in-plane and out-of-plane electronic speckle pattern interferometers," Appl. Opt., vol. 43, no. 24, pp. 4652-4658, 2004.

[2] L. Yang, X. Xie, L. Zhu, S. Wu, and Y. Wang, "Review of electronic speckle pattern interferometry (ESPI) for three dimensional displacement measurement," Chin. J. Mech. Eng., vol. 27, no. 1, pp. 1-13, Jan. 2014

[3] D. Francis, R. P. Tatam, and R. M. Groves, "Shearography technology and applications: A review," Meas. Sci. Technol., vol. 21, no. 10, Oct. 2010, Art. no. 102001

[4] Y. Y. Hung, "Applications of digital shearography for testing of composite structures," Compos. B, Eng., vol. 30, no. 7, pp. 765-773, Oct. 1999.

[5] B. Javidi and E. Tajahuerce, "Three-dimensional object recognition by use of digital holography," Opt. Lett., vol. 25, no. 9, pp. 610-612, 2000.

[6] G. Pedrini, P. Fröning, H. J. Tiziani, and F. Mendoza Santoyo, "Shape measurement of microscopic structures using digital holograms," Opt. Commun., vol. 164, nos. 4-6, pp. 257-268, Jun. 1999.

[7] P. F. Luo, Y. J. Chao, M. A. Sutton, and W. H. Peters, "Accurate measurement of three-dimensional deformations in deformable and rigid bodies using computer vision," Experim. Mech., vol. 33, no. 2, pp. 123-132, Jun. 1993.

[8] J.-J. Orteu, "3-D computer vision in experimental mechanics," Opt. Lasers Eng., vol. 47, nos. 3-4, pp. 282-291, Mar. 2009.

[9] Y. Gao, T. Cheng, Y. Su, X. Xu, Y. Zhang, and Q. Zhang, "Highefficiency and high-accuracy digital image correlation for threedimensional measurement," Opt. Lasers Eng., vol. 65, pp. 73-80, 2015.

[10] B. Pan, "Digital image correlation for surface deformation measurement: Historical developments, recent advances and future goals," Meas. Sci. Technol., vol. 29, no. 8, Aug. 2018, Art. no. 082001.

[11] L. Robert, F. Nazaret, T. Cutard, and J.-J. Orteu, "Use of 3-D digital image correlation to characterize the mechanical behavior of a fiber reinforced refractory castable," Experim. Mech., vol. 47, no. 6, pp. 761-773, Dec. 2007.

[12] M. A. Sutton, J. Yan, X. Deng, C.-S. Cheng, and P. Zavattieri, "Threedimensional digital image correlation to quantify deformation and crackopening displacement in ductile aluminum under mixed-mode i/iii loading," Opt. Eng., vol. 46, no. 5, p. 051003, 2007.

[13] N. Candau, C. Pradille, J.-L. Bouvard, and N. Billon, "On the use of a four-cameras stereovision system to characterize large 3D deformation in elastomers," Polym. Test., vol. 56, pp. 314-320, Dec. 2016.

[14] X. Shao, X. Dai, Z. Chen, and X. He, "Real-time 3d digital image correlation method and its application in human pulse monitoring," Appl. Opt., vol. 55, no. 4, pp. 696-704, 2016.

[15] D. Solav, K. M. Moerman, A. M. Jaeger, K. Genovese, and H. M. Herr, "MultiDIC: An open-source toolbox for multi-view 3D digital image correlation," IEEE Access, vol. 6, pp. 30520-30535, 2018.
[16] A. Gustafsson et al., "Linking multiscale deformation to microstructure in cortical bone using in situ loading, digital image correlation and synchrotron X-ray scattering," Acta Biomaterialia, vol. 69, pp. 323-331, Mar. 2018.

[17] C. Goidescu et al., "Damage investigation in CFRP composites using full-field measurement techniques: Combination of digital image stereocorrelation, infrared thermography and X-ray tomography," Compos. B, Eng., vol. 48, pp. 95-105, May 2013.

[18] L. Yu and B. Pan, "Experimental study of tensile properties and deformation evolutions of $2 \mathrm{D}$ and $2.5 \mathrm{D}$ woven $\mathrm{SiO}_{2} \mathrm{f} / \mathrm{SiO}_{2}$ composites using single-camera stereo-digital image correlation," Compos. Struct., vol. 200, pp. 589-598, Sep. 2018.

[19] V. Tiwari et al., "Application of 3D image correlation for full-field transient plate deformation measurements during blast loading," Int. J. Impact Eng., vol. 36, no. 6, pp. 862-874, Jun. 2009.

[20] M. N. Helfrick, C. Niezrecki, P. Avitabile, and T. Schmidt, "3D digital image correlation methods for full-field vibration measurement," Mech. Syst. Signal Process., vol. 25, no. 3, pp. 917-927, Apr. 2011.

[21] L. Yu and B. Pan, "Single-camera high-speed stereo-digital image correlation for full-field vibration measurement," Mech. Syst. Signal Process., vol. 94, pp. 374-383, Sep. 2017.

[22] M. Pankow, B. Justusson, and A. M. Waas, "Three-dimensional digital image correlation technique using single high-speed camera for measuring large out-of-plane displacements at high framing rates," Appl. Opt., vol. 49, no. 17, pp. 3418-3427, 2010.

[23] L. Yu and B. Pan, "Single-camera stereo-digital image correlation with a four-mirror adapter: Optimized design and validation," Opt. Lasers Eng., vol. 87, pp. 120-128, Dec. 2016.

[24] B. Pan, L. Yu, and Q. Zhang, "Review of single-camera stereo-digital image correlation techniques for full-field 3D shape and deformation measurement," Sci. China Technological Sci., vol. 61, no. 1, pp. 2-20, Jan. 2018.

[25] L. Yu, R. Tao, and G. Lubineau, "Accurate 3D shape, displacement and deformation measurement using a smartphone," Sensors, vol. 19, no. 3, p. 719, Feb. 2019.

[26] L. Yu and G. Lubineau, "Modeling of systematic errors in stereo-digital image correlation due to camera self-heating," Sci. Rep., vol. 9, no. 1, Dec. 2019, Art. no. 6567.

[27] J. Zhu, "Design and calibration of a single-camera-based stereo vision sensor," Opt. Eng., vol. 45, no. 8, Aug. 2006, Art. no. 083001.

[28] R. Wang, X. Li, and Y. Zhang, "Analysis and optimization of the stereosystem with a four-mirror adapter," J. Eur. Opt. Soc., Rapid, vol. 3, p. 30, Sep. 2008.

[29] Q. Ma and S. Ma, "Experimental investigation of the systematic error on photomechanic methods induced by camera self-heating," Opt. Exp., vol. 21, no. 6, pp. 7686-7698, 2013.

[30] B. Pan, W. Shi, and G. Lubineau, "Effect of camera temperature variations on stereo-digital image correlation measurements," Appl. Opt., vol. 54, no. 34, pp. 10089-10095, 2015.

[31] B. Pan, "Thermal error analysis and compensation for digital image/volume correlation," Opt. Lasers Eng., vol. 101, pp. 1-15, Feb. 2018. 